\title{
Immunostimulatory and Anti-inflammatory Effect of Ganoderma Lucidum on Breast Cancer Patients
}

\author{
Nidhal AK. Mohammed Ali'1, Hakar Abdulkareem Saeed ${ }^{1}$, Ramadhan T. Othman²
}

${ }^{1}$ Department of Pharmacology, College of Medicine, Hawler Medical University, Iraq. ${ }^{2}$ Department of Internal Medicine, College of Medicine, University of Duhok, Iraq.

\begin{abstract}
Background: Breast cancer $(\mathrm{BC})$ is the most common cancer among women. A key factor in tumour development is the evasion of immune detection, therefore; search for alternative medicines with the ability to improve the function of the immune system and targets tumours cells has received growing interest. Ganoderma lucidum (GL) has been demonstrated to possess anti-tumour and immunomodulatory activities. Little is known about the immunomodulatory effect of GL on $\mathrm{BC}$ patients when used with chemotherapeutic agents. We aim to investigate the level of immune biomarkers; interferon- $\gamma$ (IFN- $\gamma$ ), tumour necrotic factor- $\alpha$ (TNF- $\alpha$ ), interleukin-8 (IL-8) and adiponectin in these patients. Material and Methods: Forty female patients with $\mathrm{BC}$ were included and divided equally into two groups: Group 1 received chemotherapy alone and Group 2 received chemotherapy plus GL capsules (1000 mg twice daily). Blood samples were obtained from all patients before and after 4 cycles of chemotherapy. These samples were analysed to identify the levels of IFN- $\gamma$, TNF- $\alpha$, IL- 8 , and adiponectin. Results: After the treatment period, there was the significant increase in the level of IFN- $\gamma(\mathrm{P}=0.0024)$ and a significant decrease in the mean serum levels of TNF- $\alpha(\mathrm{P}=0.0004)$ and IL-8 $(\mathrm{P}=0.019)$ in patients treated with concurrent GL and chemotherapy. A non-significant difference was found between pre and post-treatment in the mean serum levels of TNF- $\alpha$, IFN- $\gamma$, and IL-8 in patients receiving chemotherapy only. The mean serum levels of adiponectin in both groups did not show any significant difference when compared to pre-treatment levels. Conclusion: The present study showed that the use of GL in-combination with chemotherapy could have immune stimulatory, anti-inflammatory and anti-metastatic activity that can enhance the therapeutic responsiveness of BC patients to chemotherapy and improve their quality of life during treatment. More research is needed to confirm these results. In addition, clinical evaluation of benefit and outcome are ongoing.
\end{abstract}

Keywords: Ganoderma lucidum- breast cancer- interferon- $\gamma$ - tumor necrotic factor- $\alpha$ - inteleukin- 8 - adiponectin

Asian Pac J Cancer Biol, 3 (2), 51-57

\section{Introduction}

Breast cancer is the most frequently diagnosed cancer and the leading cause of cancer death among females worldwide [1-2]. In Kurdistan Province/Iraq, breast cancer was reported as the most common cancer amongst females and constitutes $33.47 \%$ among all cancers [3]. Breast cancer is considered immunogenic and more than $70 \%$ of breast cancers contain lymphocytic infiltration in the stroma. Preclinical studies suggested that immune editing and partial control of cancer progression by the local immune microenvironment operate in most breast cancers [4-5]. The major modalities of breast cancer
Submission Date: 01/08/2018Ａcceptance Date: 04/11/2018

treatment to date are surgery, radiation, chemotherapy, endocrine, and immunotherapy.

There is a complex interplay among the host immune response, tumor cells, tumor microenvironment, and the effects of various treatments on these elements [6]. Numerous studies indicated that chemotherapy-associated toxicities are mediated by inflammation that may lead to resistance, failure of therapy and metastasis [7]. A variety of inflammatory mediators and signaling pathways are upregulated in response to taxanes, anthracyclines, and alkylating agents administration that are important in promoting proliferation, adhesion, resistance and metastasis of different cancer including breast cancer

Corresponding Author:

Dr. Ramadhan T. Othman

Department of Internal Medicine, College of Medicine, University of Duhok, Iraq.

Email: ramadhan.othman@uod.ac 
such as NFאB, Interleukine-1 $\beta$ (IL-1 $\beta$ ), Interleukin-8 (IL-8), Interleukin-6 (IL-6), tumour necrosis factor- $\alpha$ (TNF- $\alpha$ ), vascular endothelial growth factor-A and TollLike-Receptor-4 [8].

Extracts from a medicinal mushroom Ganoderma Lucidum (GL) have been widely used in Asian countries to treat various human diseases such as immunological disorders, inflammatory disorders, and cancer [9-10]. GL might have cytotoxic, cytostatic, anti-metastatic, anti-inflammatory, and immune-modulating activities [11].

GL contains diverse biological compounds, including polysaccharides that stimulate the immune system and triterpenes that exhibit cytotoxicity against cancer cells [12]. It has been demonstrated that GL stimulates $\mathrm{B}$ cell proliferation and activation, promotes $\mathrm{T}$ cell release of interferon- $\gamma$ (IFN- $\gamma$ ), enhances activation and maturation of immature dendritic cells promotes macrophage differentiation and maturation and sensitized NK cell-mediated cytotoxicity [11].

Ganoderic acid, an active ingredient of GL, displays potent anti-angiogenesis activity, via suppression of proliferation of endothelial cells [13]. Numerous pharmacological and clinical investigations demonstrated that along with immunomodulatory activities, the anti-tumour of GL had raised the possibility that it could be effective in the cancer patients receiving conventional chemotherapy and/or radiation treatment, to inhibit immune resistance and reduce chemotherapy toxicities [14-16].

GL has shown anti-tumour activity in vitro and in vivo studies [17-18]. The effects of GL were tested in phase I and phase II trial in advance cancer patients. These studies showed that advanced lung and colon cancer patients treated with GL showed improvement in immune responses [19-20]. However, patients with advanced stage disease have the short life expectancy and suffer from end-stage diseases. Additionally, they have used GL as the single agent without chemotherapy and they did not include any control group. The purposes of our study were to investigate specific effects of GL on some immunological, inflammatory and other markers involved in the proliferation, angiogenesis and metastasis of breast cancer patients such as IFN- $\gamma$, TNF- $\alpha$, IL- 8 and adiponectin (ADN) in patients treated with chemotherapy and GL compared to chemotherapy alone as a control group.

\section{Materials and Methods}

\section{Patients}

This prospective observational study was conducted at the department of pharmacology, College of medicine, Hawler Medical University in cooperation with the University of Duhok and Duhok General Directorate of Health, Oncology Department in Azadi Teaching Hospital and Nanakaly Hospital.

All patients' or their carer participating in this study signed informed consent to receive chemotherapy with and without GL, in addition, they agreed on blood sample collection and to have their data collected and used for research. Duhok General Directorate of Health Ethics Committee and both hospitals approved this study. In this prospective analysis, 40 female with $\mathrm{BC}$ were included (Table 1).

All patients received treatment at Haemato-oncology department/ Azadi Teaching Hospital and Nanakaly hospital from September 2016-to December 2017. A quasi-experimental design was used and patients were divided into 2 equal groups in terms of treatment type that they had been given. The first Group receives chemotherapy alone (control). The second Group was treated with chemotherapy plus GL under the supervision of medical oncologist for 12 weeks (four chemotherapy cycle) after explaining the role and side effects of GL to each patient. Patients were recruited into the study if they met following eligibility criteria: Female with confirmed breast cancer (stage II-IV). ECOG 0-2. Adequate bone marrow, renal and liver functions. Projected life expectancy of $>12$ weeks. Patients were excluded if they have brain metastasis and patients with liver, kidney impairment, and cardiac insufficiency. All patients included in this study were female and were treated after surgery with various chemotherapy protocols (mainly doxorubicin in combination with cyclophosphamide) 37 patients were assessable for immune function after 12 weeks of treatments. Three patients were not assessed and excluded from the study due to loss of follow up by two of them non-compliance by another one.

\section{Drug administration}

The GL capsule was the therapeutic agent administered during 12-week of the study period. Patients were treated with $1000 \mathrm{mg}$ capsule 2 times daily orally after meals ((NATURE'S ANSWERCNY, USA; the whole plant, $1000 \mathrm{mg}$ contain 90 capsules per container).

\section{Determination of Plasma Cytokine Concentrations}

Blood samples (plasma) were obtained from patients before and after treatment and were assayed for the determination of IFN- $\gamma$ (Bioassay Technology Laboratory, Korea), TNF- $\alpha$, IL-8, and adiponectin using commercially available ELISA kits (EASTBIOPHARM, USA). Assay procedures were done according to manufacturer's instructions. All determinations were performed in triplicate.

\section{Statistical analysis}

The changes in the level of immune markers were assessed before and after treatment in both Groups 1 and 2. Data were analysed using the analysis of variance, all data expressed as Mean \pm SD. SPSS version 19 was used, differences between 2 groups were determined using Student's t-test. In all tests, $\mathrm{P}$ values of $<0.05$ were considered significant.

\section{Results}

Patient characteristics

All patients were divided into 2 group each group 
includes 20 patients. The mean age for Group 1 at time of diagnosis was $45.9 \pm 8.25$ years, while the mean age for Group 2 was $47.4 \pm 13.73$ years. (Table1). The mean body mass index (BMI) of patients in Group 1 and Group 2 was $28.49 \pm 5.97$ and $27.64 \pm 5.45$ respectively (Table 1).

We started to select cases according to their presentation as the first group treated with chemotherapy alone (control group), the second groups of patients were treated with chemotherapy and GL and they were selected after completing the first group. All cases were classified into four molecular subtypes according to immunohistochemistry results for ER, PR, Her-2 and Ki67. They have been classified according to guideline into luminal A, luminal B, Her-2 positive and triple negative (Table 2).

\section{Changes in plasma concentration of IFN- $\gamma$}

The serum level of IFN- $\gamma$ was measured in Group 1 control group who were treated with chemotherapy and Group 2 patients who were treated with chemotherapy and GL. The changes in the concentration of IFN- $\gamma$ were measured before and after treatment for 12 weeks in both groups. A Non-significant $(\mathrm{P} \geq 0.374)$ difference was found in the mean of serum IFN- $\gamma$ levels in patients of Group 1 before and after chemotherapy administration $(13.26 \pm 0.75 \mathrm{ng} / \mathrm{ml})$ and $(11.43 \pm 0.37 \mathrm{ng} / \mathrm{ml})$ respectively (Table 3). While in Group 2 patients the mean serum levels of IFN- $\gamma$ increased significantly $(\mathrm{P} \leq 0.0024)$ after administration of GL with chemotherapy from $(11.54 \pm 0.39 \mathrm{ng} / \mathrm{ml})$ to $(13.24 \pm 0.36 \mathrm{ng} / \mathrm{ml})$ (Table 4).

\section{Changes in plasma concentration of TNF- $\alpha$}

The TNF- $\alpha$ was measured in Group 1 and Group 2 before and after treatment. No significant changes were observed in the level of TNF- $\alpha$ in Group 1 before and after administration of chemotherapy ( $\mathrm{P} \geq 0.581$ ) (Table 3).
While in Group 2, there was a significant reduction (P $\leq 0.0004)$ in the mean serum levels of TNF- $\alpha$ after administration of GL with chemotherapy $(267.82 \pm 14.78$ $\mathrm{ng} / \mathrm{L})$ compared to those before chemotherapy treatment $(360.89 \pm 28.93 \mathrm{ng} / \mathrm{L})$ (Table 4).

\section{Changes in plasma concentration of IL-8}

There was non-significantly increased after administration of chemotherapy from $(247.05 \pm 26.1$ $\mathrm{ng} / \mathrm{L})$ to $(288.71 \pm 32.9 \mathrm{ng} / \mathrm{L})(\mathrm{P} \geq 0.112)$ (Table 3.3). However, a significant reduction was observed in the mean serum levels of IL-8 after administration of GL with chemotherapy $(252.63 \pm 36.0 \mathrm{ng} / \mathrm{L})$ compared to the level before treatment $(360.4 \pm 62.58 \mathrm{ng} / \mathrm{L})(\mathrm{P} \leq 0.019)$ (Table 4) (Figure 1).
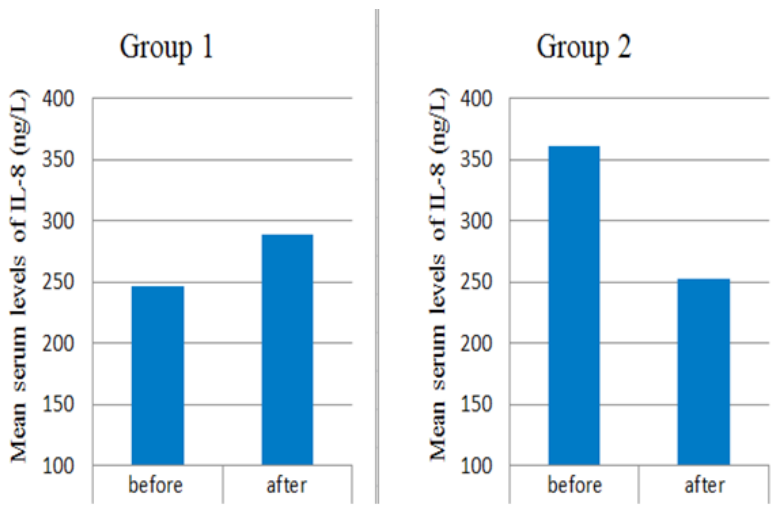

Figure 1. Serum Level Changes of IL-8 in Group 1 Treated Patients with Chemotherapy and Group 2 Treated with Chemotherapy Ganoderma Lucidum. Significant reduction of volume was observed in group 1 compared to control group $(\mathrm{P}=0.004)$.

Table 1. Demographic Characteristics and Clinical Data of Patients in Study Groups

\begin{tabular}{lccc}
\hline & Group 1 $(\mathrm{n}=20)$ & Group 2 $(\mathrm{n}=20)$ & P value \\
\hline Age & $45.9 \pm 8.25$ & $47.4 \pm 13.73$ & 0.675 \\
BMI & $28.49 \pm 5.97$ & $27.64 \pm 5.45$ & 0.640 \\
Stage of cancer: & 6 & 5 & \\
$\quad$ Stage II & 12 & 12 (one inflammatory breast cancer) & \\
Stage III & 2 & 3 & \\
Stage IV & & & \\
\hline
\end{tabular}

Group 1 are patients treated with chemotherapy while Group 2 are patients treated with chemotherapy and Ganoderma Lucidum. BMI, Body mass index; TNBC, Triple negative breast cancer

Table 2. Molecular Subtypes

\begin{tabular}{lccc}
\hline molecular subtypes & group 1 No.(\%) & group 2 No.(\%) & both group No.(\%) \\
\hline luminal A & $10(50)$ & $5(25)$ & $15(37.5)$ \\
luminal B & $4(20)$ & $7(35)$ & $11(27.5)$ \\
HER 2 enriched & $2(10)$ & $5(25)$ & $7(12.5)$ \\
Basal like & $4(20)$ & $3(15)$ & $7(17.5)$ \\
Total & $20(100)$ & $20(100)$ & $40(100)$ \\
\hline
\end{tabular}

Luminal A, ER and/or PR +ve, Her2 -ve; Luminal B, ER and/or PR +ve Her2 -ve but Ki67>20\% ER and/or PR +ve; Her2 +ve, Her2; ER, PR -ve Her2 +ve, Basla like, ER, PR, Her2 -ve 
Table 3. Mean Serum Levels of Interferon- $\gamma$ (IFN- $\gamma$ ); Tumor Necrosis Factor- $\alpha$ (TNF- $\alpha$ ); Interlukin-8 (IL-8) and Adiponectin $(\mathrm{ADN})$ in the Patients $(\mathrm{n}=20)$ before and after Receiving Chemotherapy Alone . P value $\leq 0.05$ Considered Statistically Significant

\begin{tabular}{lccc}
\hline Biomarker & Before Treatment & After Treatment & P value \\
\hline IFN- $\gamma(\mathrm{ng} / \mathrm{ml})$ & $13.26 \pm 1.97$ & $11.43 \pm 0.36$ & 0.374 \\
$\mathrm{TNF}-\alpha(\mathrm{ng} / \mathrm{L})$ & $353.59 \pm 67.82$ & $317.8 \pm 19.70$ & 0.581 \\
$\mathrm{IL}-8(\mathrm{ng} / \mathrm{L})$ & $247.05 \pm 26.1$ & $288.71 \pm 32.9$ & 0.112 \\
$\mathrm{ADN}(\mathrm{mg} / \mathrm{L})$ & $9.61 \pm 0.68$ & $9.72 \pm 0.60$ & 0.857 \\
\hline
\end{tabular}

Table 4. Mean Serum Levels of Interferon- $\gamma$ (IFN- $\gamma$ ); Tumor Necrosis Factor- $\alpha$ (TNF- $\alpha$ ); Interlukin-8 (IL-8) and Adiponectin $(\mathrm{ADN})$ in Patients $(\mathrm{n}=20)$ before and after Receiving Ganoderma Lucidum with Chemotherapy. $P$ value $\leq 0.05$ Considered Statistically Significant

\begin{tabular}{lccc}
\hline Biomarker & Before Treatment & After Treatment & P value \\
\hline IFN- $\gamma(\mathrm{ng} / \mathrm{ml})$ & $11.54 \pm 0.39$ & $13.24 \pm 0.36$ & 0.0024 \\
$\mathrm{TNF}-\alpha(\mathrm{ng} / \mathrm{L})$ & $360.89 \pm 28.93$ & $267.82 \pm 14.78$ & 0.0004 \\
$\mathrm{IL}-8(\mathrm{ng} / \mathrm{L})$ & $360.4 \pm 62.58$ & $252.63 \pm 36.0$ & 0.0190 \\
$\mathrm{ADN}(\mathrm{mg} / \mathrm{L})$ & $9.88 \pm 0.94$ & $9.76 \pm 0.88$ & 0.9802 \\
\hline
\end{tabular}

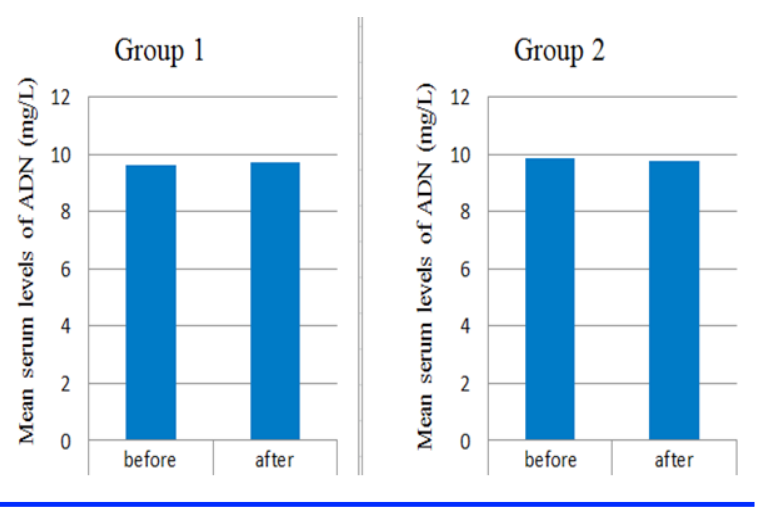

Figure 2. Serum Level Changes of Adiponectin in Group 1 Treated Patients with Chemotherapy and Group 2 Treated with Chemotherapy Ganoderma Lucidum. Significant reduction of volume was observed in group1 compared to control group $(\mathrm{P}=0.004)$.

\section{Changes in plasma concentration of Adiponectin}

There was a non-significant $(\mathrm{P} \leq 0.857)$ difference in the mean serum levels of Adiponectin in Group 1 patients after administration of chemotherapy $(9.72 \pm 0.60 \mathrm{mg} / \mathrm{L})$ compared to the mean serum levels of $(9.61 \pm 0.68 \mathrm{mg} / \mathrm{L})$ before chemotherapy administration (Table 3 and Figure 2). Also, a non-significant $(\mathrm{P} \geq 0.9802)$ difference was observed in the mean serum levels of adiponectin after treatment with GL plus chemotherapy $(9.86 \pm 0.86 \mathrm{mg} / \mathrm{L})$ compared to the level before treatment $(9.83 \pm 0.93 \mathrm{mg} / \mathrm{L})$ (Table 4 and Figure 2$)$.

\section{Discussion}

To the best of our knowledge, this study is the first to evaluate the effect of GL on immune-modulation in advance breast cancer $(\mathrm{ABC})$ patients. The study confirms that the use of GL in ABC patients treated with chemotherapy can improve their immunity. Analysis of immune markers in $20 \mathrm{BC}$ patients revealed that IFN- $\gamma$, TNF- $\alpha$, and IL-8 significantly changed over 12 weeks after starting treatment with GL plus chemotherapy compared to control patients treated with chemotherapy alone.

By using patients treated with chemotherapy alone as a 'control' group, the study attempted to control for the potential direct impact of chemotherapy on these immune markers. Therefore, this supports that our findings are not related to the effect of chemotherapy on immunity but they are rather due to the effect of GL when used concurrently with chemotherapy.

Evidence in BC patient treated with chemotherapy has indicated that the immune function can be reduced or damaged resulting in a decrease in cytotoxic T lymphocyte and NK activity. Additionally, in patients with advanced stage BC, an impaired immune function was observed that promote tumour progression and metastasis [21]. Therefore, standard chemotherapy might reduce the therapeutic benefits obtained by the increased tumour killing of the treatment. These negative effects may be particularly important in the treatment of immunogenic tumours, where the immunological function of the host is a determining factor for the clinical outcome of treatment. Thus, a combination of chemotherapy with immune-modulating agents may provide a strategy for overcoming the immunosuppressive effects of chemotherapy. Results from this study showed the significant increase in the level of IF- $\gamma$ in patients treated with GL plus chemotherapy indicate its ability to trigger an immunostimulatory response and activation of innate immune cells required for promoting cytostatic/cytotoxic and antitumor mechanisms during the cell-mediated adaptive immune response. The increased IFN- $\gamma$ can contribute to the containment of tumour progression and growth by increasing tumour antigen presentation to a tumour specific T cells and increase susceptibility to NK cytotoxicity. In addition to promoting the immune response to a tumour, IFN- $\gamma$ also can induce the expression of a tumour suppressing factors. The findings of the current study were consistent with the finding of previous studies on advanced-stage cancer patients that treated where they 
showed a significant increase in IFN- $\gamma$ level in patients treated with GL [22].

Recently, many biological response modifiers have been combined with cytotoxic chemotherapeutic agents, an attempt to enhance anti-cancer activity and reduce toxicity. As many herbal medicines appear to work as biological response modifiers, they may become an important complementary approach to cancer treatment. These are agents or approaches modifying the relationship between the host and tumour by activating, increasing, and/or restoring the reactivity of immunological effector mechanisms that are involved in resistance to tumour growth and metastasis, leading to therapeutic effects [22-23]. Our study indicate that GL significantly reduce TNF- $\alpha$, pro-inflammatory cytokine, which is shown to be up-regulated in breast cancer and known to promote the growth, proliferation of breast cancer cells, expression of adhesion molecules and facilitating the invasion of metastatic tumour cells and through this effect GL would possibly augment the activity of chemotherapy [24]. Moreover, the significant reduction in TNF- $\alpha$ observed in the current study may contribute to inhibiting metastasis of breast cancer to the bone through inhibiting osteoclastogenesis since TNF- $\alpha$ shown to stimulate osteoclastic response (25]. Reduction of TNF- $\alpha$ was correlated improving fatigability in $\mathrm{BC}$ patient treated with endocrine therapy [26]. In addition, the inhibitory effects of GL on TNF- $\alpha$ production may result in other beneficial effects such as improving quality of life in cancer patients. Increased TNF- $\alpha$ has been thought to contribute to cancer cachexia that is manifested by bodyweight loss, chronic nausea, fatigue, insomnia and profuse sweating [27-28]. Drugs that down-regulate TNF-a can result in improvements of features related to weight loss and cachexia [29]. Thus, GL may represent a useful approach to improve cancer patients quality of life and inhibit cancer or treatment-related cachexia.

The interleukin-8 expression has been shown to be regulated by a number of different stimuli including inflammatory signals (e.g., TNF- $\alpha$, IL-1 $\beta$ ), exposure to chemotherapy agents, hypoxia, and steroid hormones such as androgens, estrogens, and dexamethasone [30]. IL-8 and its ligands CXCR $1 / 2$ are important in facilitating the metastatic colonization of breast cancer and higher IL-8 levels at metastatic sites were reported in clinical studies compared with primary sites [31]. In vitro study showed that GL inhibits oxidative stress-induced phosphorylation and inhibition of breast cancer cells migration by suppression of IL-8 secretion [32]. Patients received GL during chemotherapy showed a significant decrease in serum level of IL-8 is in agreement with studies that demonstrate down-regulation of IL- 8 expression by GL in tubular epithelial cells exposed to oxidative damage [33]. Thus inhibition or down-regulation IL-8 that was observed in our study implies that GL has the ability to suppress this proinflammatory chemokine and would most likely inhibit migration of breast cancer cells and metastasis and sensitize breast cancer cells to the cytotoxic effects of chemotherapeutic agents [34].

The serial assessment of immune markers (IFN- $\gamma$,
TNF- $\alpha$, and IL-8) in patients treated with chemotherapy alone showed no significant changes in their level before and after chemotherapy which again support the importance of the use of GL in BC patients treated with chemotherapy [21-35].

This study indicates that GL has stimulated host defense response, through modulating of different immune markers but the mechanism for these effects of GL is unclear. However previous studies showed that polysaccharides $\beta$-glucans, GL triterpene are the main extract that has anti-inflammatory and antitumor activity [36-37]. Further studies are needed to investigate which substance in GL might have the potent immune-stimulating effects and the mechanisms involved.

This study could not find a difference in the level of serum adiponectin. Several studies demonstrated that high adiponectin levels promote anti-inflammatory and immunomodulation effects [38] and various observational studies of epidemiologic cohorts stated that serum adiponectin is inversely correlated to the development of various cancers [39-40]. The non-significant elevation of adiponectin in both studied groups could be due to either: 1) Patients' mean BMI that was not $\geq 30$. 2) The small sample size of patients in both studied groups that did not result in a significant increment in adiponectin serum levels. 3) Higher doses of GL more than ( $2 \mathrm{~g} /$ day) is needed or it might need to be given for a longer duration [41].

Our results showed that GL in combination with chemotherapy can significantly increase serum level of immune markers including IFN- $\gamma$, TNF- $\alpha$, and IL-8 whereas no changes were observed in the adiponectin level. Given that these markers play an important role in enhancing immune activity, there may be the important clinical implication for use of GL in combination with chemotherapy in $\mathrm{ABC}$ patients.

In conclusion, based on our findings, we conclude that GL use with chemotherapy can have immune stimulatory, anti-inflammatory and anti-metastatic activity. These results open new opportunities for further studies to investigate the efficacy of GL in combination with chemotherapy. These findings strongly suggest that GL used in combination with chemotherapy may act as a host defense potentiator that might enhance the therapeutic responsiveness of $\mathrm{BC}$ patients to chemotherapy and improve their quality of life during treatment. Clinical evaluations of response and toxicity are ongoing.

\section{Acknowledgments}

The authors gratefully appreciate the support by the Hawler Medical College/ Hawler University, Duhok Medical College/ the University of Duhok, haematooncology department/ Azadi Teaching Hospital and Nanakaly hospital Corporation, Kurdistan, Iraq.

\section{References}

1. Torre LA, Bray F, Siegel RL, Ferlay J, Lortet-Tieulent J, Jemal A. Global cancer statistics, 2012. CA: a cancer journal for 
clinicians. 2015;65(2):87-108.

2. Siegel RL, Miller KD, Jemal A. Cancer statistics, 2016. CA: a cancer journal for clinicians. 2016;66(1):7-30.

3. Othman RT, Abdulljabar R, Saeed A, Kittani SS, Sulaiman HM, Mohammed SA, et al. Cancer incidence rates in the Kurdistan region/Iraq from 2007-2009. Asian Pacific journal of cancer prevention : APJCP. 2011;12(5):1261-4.

4. Pusztai L, Karn T, Safonov A, Abu-Khalaf MM, Bianchini G. New Strategies in Breast Cancer: Immunotherapy. Clinical cancer research : an official journal of the American Association for Cancer Research. 2016;22(9):2105-10.

5. Wang M, Zhang C, Song Y, Wang Z, Wang Y, Luo F, et al. Mechanism of immune evasion in breast cancer. OncoTargets and therapy. 2017;10:1561-73.

6. Bracci L, Schiavoni G, Sistigu A, Belardelli F. Immunebased mechanisms of cytotoxic chemotherapy: implications for the design of novel and rationale-based combined treatments against cancer. Cell death and differentiation. 2014;21(1):15-25.

7. Vichaya EG, Chiu GS, Krukowski K, Lacourt TE, Kavelaars A, Dantzer R, et al. Mechanisms of chemotherapy-induced behavioral toxicities. Frontiers in neuroscience. 2015;9:131.

8. Vyas D, Laput G, Vyas AK. Chemotherapy-enhanced inflammation may lead to the failure of therapy and metastasis. OncoTargets and therapy. 2014;7:1015-23.

9. Jiang J, Sliva D. Novel medicinal mushroom blend suppresses growth and invasiveness of human breast cancer cells. International journal of oncology. 2010;37(6):1529-36.

10. Bishop KS, Kao CH, Xu Y, Glucina MP, Paterson RR, Ferguson LR. From 2000years of Ganoderma lucidum to recent developments in nutraceuticals. Phytochemistry. 2015;114:56-65.

11. Xu Z, Chen X, Zhong Z, Chen L, Wang Y. Ganoderma lucidum polysaccharides: immunomodulation and potential anti-tumor activities. The American journal of Chinese medicine. 2011;39(1):15-27.

12. Martinez-Montemayor MM, Acevedo RR, Otero-Franqui E, Cubano LA, Dharmawardhane SF. Ganoderma lucidum (Reishi) inhibits cancer cell growth and expression of key molecules in inflammatory breast cancer. Nutrition and cancer. 2011;63(7):1085-94.

13. Tung NT, Cuong TD, Hung TM, Lee JH, Woo MH, Choi JS, et al. Inhibitory effect on NO production of triterpenes from the fruiting bodies of Ganoderma lucidum. Bioorganic \& medicinal chemistry letters. 2013;23(5):1428-32.

14. Batbayar S, Kim MJ, Kim HW. Medicinal mushroom Lingzhi or Reishi, Ganoderma lucidum (W.Curt.:Fr.) P. Karst., beta-glucan induces Toll-like receptors and fails to induce inflammatory cytokines in NF-kappaB inhibitor-treated macrophages. International journal of medicinal mushrooms. 2011;13(3):213-25.

15. Guggenheim AG, Wright KM, Zwickey HL. Immune Modulation From Five Major Mushrooms: Application to Integrative Oncology. Integrative medicine (Encinitas, Calif). 2014;13(1):32-44.

16. Wu GS, Guo JJ, Bao JL, Li XW, Chen XP, Lu JJ, et al. Anticancer properties of triterpenoids isolated from Ganoderma lucidum - a review. Expert opinion on investigational drugs. 2013;22(8):981-92.

17. Yue QX, Xie FB, Guan SH, Ma C, Yang M, Jiang BH, et al. Interaction of Ganoderma triterpenes with doxorubicin and proteomic characterization of the possible molecular targets of Ganoderma triterpenes. Cancer science. 2008;99(7):146170 .

18. Thyagarajan A, Jedinak A, Nguyen H, Terry C, Baldridge LA, Jiang J, et al. Triterpenes from Ganoderma Lucidum induce autophagy in colon cancer through the inhibition of p38 mitogen-activated kinase (p38 MAPK). Nutrition and cancer. 2010;62(5):630-40.

19. Chen X, Hu ZP, Yang XX, Huang M, Gao Y, Tang W, et al. Monitoring of immune responses to a herbal immunomodulator in patients with advanced colorectal cancer. International immunopharmacology. 2006;6(3):499-508.

20. Gao Y, Tang W, Dai X, Gao H, Chen G, Ye J, et al. Effects of water-soluble Ganoderma lucidum polysaccharides on the immune functions of patients with advanced lung cancer. Journal of medicinal food. 2005;8(2):159-68.

21. Critchley-Thorne RJ, Simons DL, Yan N, Miyahira AK, Dirbas FM, Johnson DL, et al. Impaired interferon signaling is a common immune defect in human cancer. Proceedings of the National Academy of Sciences of the United States of America. 2009;106(22):9010-5.

22. Gao Y, Zhou S, Jiang W, Huang M, Dai X. Effects of ganopoly (a Ganoderma lucidum polysaccharide extract) on the immune functions in advanced-stage cancer patients. Immunological investigations. 2003;32(3):201-15.

23. Zhou XL, Fan W, Yang G, Yu MX. The clinical significance of PR, ER, NF- kappa B, and TNF- alpha in breast cancer. Disease markers. 2014;2014:494581.

24. Korobeinikova E, Myrzaliyeva D, Ugenskiene R, Raulinaityte $\mathrm{D}$, Gedminaite J, Smigelskas K, et al. The prognostic value of IL10 and TNF alpha functional polymorphisms in premenopausal early-stage breast cancer patients. BMC genetics. 2015; 16:70.

25. Jiang J, Grieb B, Thyagarajan A, Sliva D. Ganoderic acids suppress growth and invasive behavior of breast cancer cells by modulating AP-1 and NF-kappaB signaling. International journal of molecular medicine. 2008;21(5):577-84.

26. Zhao H, Zhang Q, Zhao L, Huang X, Wang J, Kang X. Spore Powder of Ganoderma lucidum Improves CancerRelated Fatigue in Breast Cancer Patients Undergoing Endocrine Therapy: A Pilot Clinical Trial. Evidencebased complementary and alternative medicine : eCAM. 2012;2012:809614.

27. Argiles JM, Lopez-Soriano FJ. The role of cytokines in cancer cachexia. Medicinal research reviews. 1999;19(3):223-48.

28. Mendes MC, Pimentel GD, Costa FO, Carvalheira JB. Molecular and neuroendocrine mechanisms of cancer cachexia. The Journal of endocrinology. 2015;226(3):R2943.

29. Haslett PA. Anticytokine approaches to the treatment of anorexia and cachexia. Seminars in oncology. 1998;25(2 Suppl 6):53-7.

30. Waugh DJ, Wilson C. The interleukin-8 pathway in cancer. Clinical cancer research : an official journal of the American Association for Cancer Research. 2008;14(21):6735-41.

31. Bieche I, Chavey C, Andrieu C, Busson M, Vacher S, Le Corre $\mathrm{L}$, et al. CXC chemokines located in the $4 \mathrm{q} 21$ region are up-regulated in breast cancer. Endocrine-related cancer. 2007;14(4):1039-52.

32. Thyagarajan A, Jiang J, Hopf A, Adamec J, Sliva D. Inhibition of oxidative stress-induced invasiveness of cancer cells by Ganoderma lucidum is mediated through the suppression of interleukin-8 secretion. International journal of molecular medicine. 2006;18(4):657-64.

33. Lai KN, Chan LY, Tang SC, Leung JC. Ganoderma extract prevents albumin-induced oxidative damage and chemokines synthesis in cultured human proximal tubular epithelial cells. Nephrology, dialysis, transplantation : official publication of the European Dialysis and Transplant Association - European Renal Association. 2006;21(5):1188-97.

34. Shi Z, Yang WM, Chen LP, Yang DH, Zhou Q, Zhu J, et al. Enhanced chemosensitization in multidrug-resistant human 
breast cancer cells by inhibition of IL- 6 and IL- 8 production. Breast cancer research and treatment. 2012;135(3):737-47.

35. Derin D, Soydinc HO, Guney N, Tas F, Camlica H, Duranyildiz D, et al. Serum IL-8 and IL-12 levels in breast cancer. Medical oncology (Northwood, London, England). 2007;24(2):163-8.

36. Dudhgaonkar S, Thyagarajan A, Sliva D. Suppression of the inflammatory response by triterpenes isolated from the mushroom Ganoderma lucidum. International immunopharmacology. 2009;9(11):1272-80.

37. Zhu XL, Chen AF, Lin ZB. Ganoderma lucidum polysaccharides enhance the function of immunological effector cells in immunosuppressed mice. Journal of ethnopharmacology. 2007;111(2):219-26.

38. Ohashi K, Parker JL, Ouchi N, Higuchi A, Vita JA, Gokce N, et al. Adiponectin promotes macrophage polarization toward an anti-inflammatory phenotype. The Journal of biological chemistry. 2010;285(9):6153-60.

39. Tworoger SS, Eliassen AH, Kelesidis T, Colditz GA, Willett WC, Mantzoros CS, et al. Plasma adiponectin concentrations and risk of incident breast cancer. The Journal of clinical endocrinology and metabolism. 2007;92(4):1510-6.

40. Shahar S, Salleh RM, Ghazali AR, Koon PB, Mohamud WN. Roles of adiposity, lifetime physical activity and serum adiponectin in occurrence of breast cancer among Malaysian women in Klang Valley. Asian Pacific journal of cancer prevention : APJCP. 2010;11(1):61-6.

41. Matthews SB, Thompson HJ. The Obesity-Breast Cancer Conundrum: An Analysis of the Issues. International journal of molecular sciences. 2016;17(6).

This work is licensed under a Creative Commons AttributionNon Commercial 4.0 International License. 